Supporting Information

\title{
How to Fabricate a Surface-Grafted Polythiophene on H-Si(100)2×1 \\ Surface via Self-Assembling and In Situ Surface Polymerization? - A Theoretical Guide
}

\author{
Xiaojing Yao, ${ }^{1}$ Jinlan Wang, ${ }^{1,2,} *$ Gang $W u,{ }^{3}$ Jianwei $X u^{4}$ and Shuo-Wang Yang ${ }^{3, *}$ \\ ${ }^{1}$ Department of Physics, Southeast University, Nanjing 211189, China \\ ${ }^{2}$ Synergetic Innovation Center for Quantum Effects and Applications (SICQEA), Hunan \\ Normal University, Changsha 410081, China \\ ${ }^{3}$ Institute of High Performance Computing, Agency for Science, Technology and Research, 1 \\ Fusionopolis Way, \#16-16 Connexis, Singapore 138632, Republic of Singapore \\ ${ }^{4}$ Institute of Materials Research and Engineering, Agency for Science, Technology and \\ Research, 2 Fusionopolis Way, Innovis, \#08-03, Singapore 138634, Republic of Singapore
}


(a)

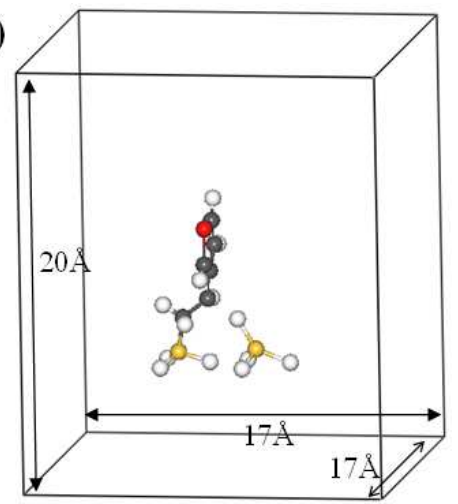

(c)

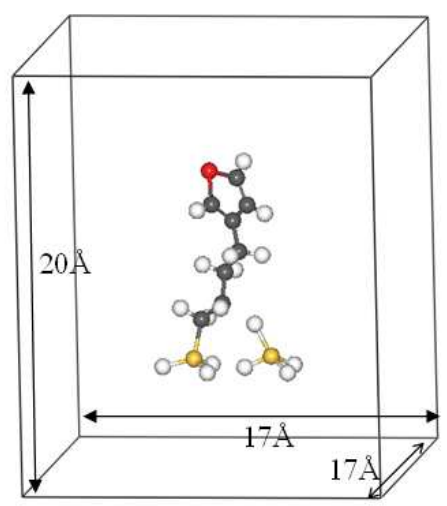

(b)

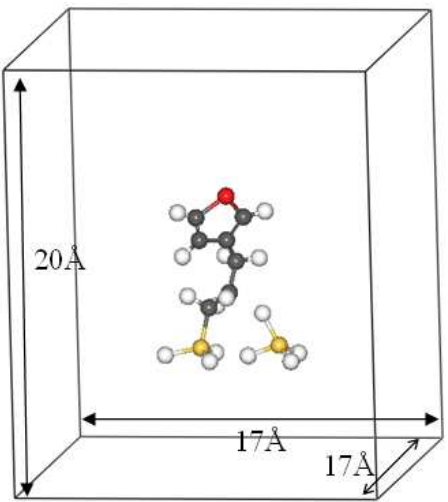

(d)

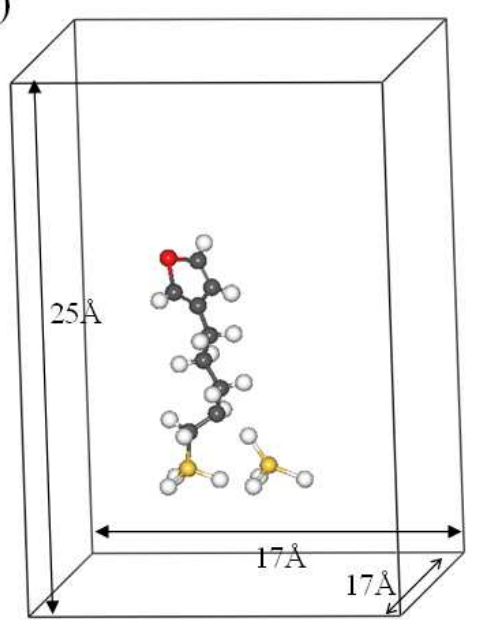

Figure S1. Cluster model used for transition state location for molecular growth reaction. The fixed $\mathrm{SiH}_{3} / \mathrm{GeH}_{3}$ groups were used instead of superlattice surfaces. 
(a)

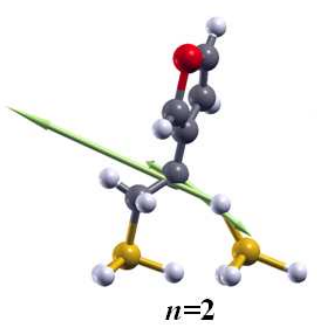

(e)

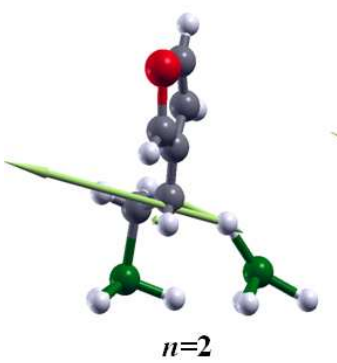

(b)

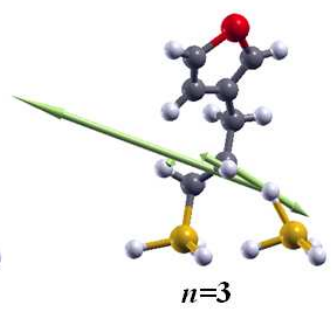

(f)

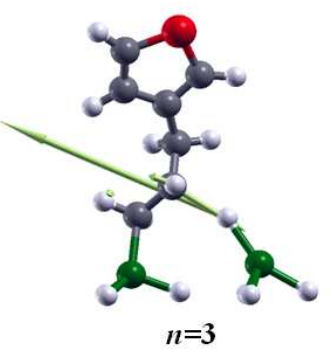

(c)

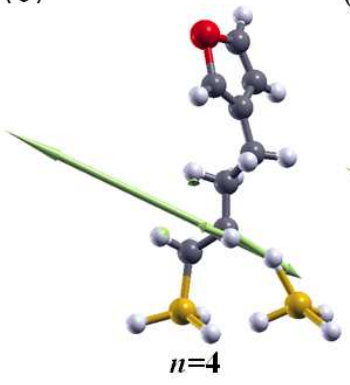

(g)

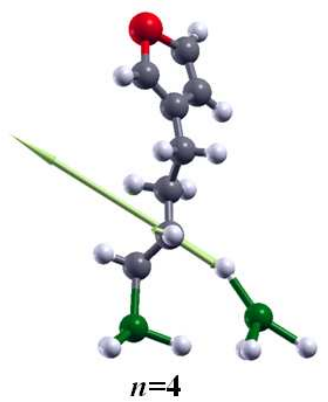

(d)

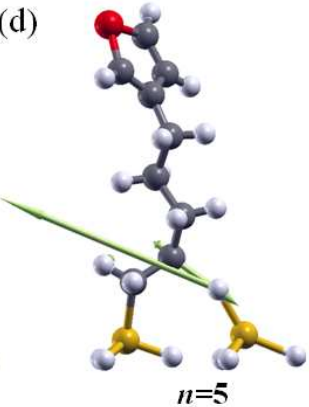

(h)

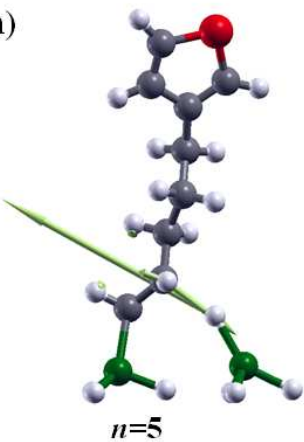

Figure S2. (a-d): the soft mode corresponding to the transition state for different $n$ on $\mathrm{Si}(100)-2 \times 1$ surfaces; and (e-h): $\mathrm{Ge}(100)-2 \times 1$ surface.

Table S1. The average adsorption energies after $1^{\text {st }}, 2^{\text {nd }}, 3^{\text {rd }} \mathrm{H}_{2} \mathrm{C}=\mathrm{CH}-\left(\mathrm{CH}_{2}\right)_{n}$-thiophene adsorptions and molecular array formations for heterolateral configuration when $n=0 \sim 3$ on silicon surface using $4 \times 2$ supercell. The Eads- $1^{\text {st }}, 2^{\text {nd }}, 3^{\text {rd }}$ and Eads-array represent the average adsorption energies after $1^{\text {st }}, 2^{\text {nd }}, 3^{\text {rd }}$ molecules and average adsorption energy of molecular array, respectively.

\begin{tabular}{ccccc}
\hline \hline & \multicolumn{4}{c}{ On Si(100)-2 $\times \mathbf{1}(\mathrm{eV})$} \\
\cline { 2 - 5 } $\boldsymbol{n}$ & 0 & 1 & 2 & 3 \\
\hline Eads-1 $^{\text {st }}$ & 1.06 & 1.19 & 1.16 & 1.12 \\
Eads-2 $^{\text {nd }}$ & 0.93 & 1.09 & 1.11 & 1.08 \\
Eads-3 $^{\text {rd }}$ & 0.94 & 1.06 & 1.09 & 1.07 \\
Eads-array & 0.91 & 1.03 & 1.07 & 1.01 \\
\hline
\end{tabular}

